\title{
On hyper-dual generalized Fibonacci numbers
}

\author{
Neşe Ömür ${ }^{1}$ and Sibel Koparal ${ }^{2}$ \\ ${ }^{1}$ Department of Mathematics, University of Kocaeli \\ 41380 İzmit, Kocaeli, Turkey \\ e-mail: neseomur@gmail.com \\ ${ }^{2}$ Department of Mathematics, University of Kocaeli \\ 41380 İzmit, Kocaeli, Turkey \\ e-mail: sibel.koparal@kocaeli.edu.tr
}

Received: 3 June 2019

Revised: 26 December 2019

Accepted: 8 January 2020

Abstract: In this paper, we define hyper-dual generalized Fibonacci numbers. We give the Binet formulae, the generating functions and some basic identities for these numbers.

Keywords: Second order linear recurrence, Hyper-dual generalized Fibonacci number.

2010 Mathematics Subject Classification: 11B50, 11A07, 11B68.

\section{Introduction}

The Fibonacci numbers was introduced by Leonardo (1170-1240) in his book Liber Abbaci that was published in 1202. Fibonacci and Lucas numbers have recently been studied by many authors.

The generalized second order sequences $\left\{U_{n}\right\}$ and $\left\{V_{n}\right\}$, are defined for $n>0$ and nonzero integer number $p$ by

$$
U_{n+1}=p U_{n}+U_{n-1} \text { and } V_{n+1}=p V_{n}+V_{n-1},
$$

in which $U_{0}=0, U_{1}=1$ and $V_{0}=2, V_{1}=p$, respectively.

The Binet formulae are

$$
U_{n}=\frac{\alpha^{n}-\beta^{n}}{\alpha-\beta} \text { and } V_{n}=\alpha^{n}+\beta^{n}
$$

where $\alpha, \beta=\left(p \pm \sqrt{p^{2}+4}\right) / 2$. 
Especially, when $p=1$, then $U_{n}=F_{n}$ (the $n$-th Fibonacci number) and $V_{n}=L_{n}$ (the $n$-th Lucas number).

In [7], E. Kılıç and P. Stanica derived the following recurrence relations for the sequences $\left\{U_{k n}\right\}$ and $\left\{V_{k n}\right\}$. For $k \geq 0, n>0$,

$$
U_{k(n+1)}=V_{k} U_{k n}+(-1)^{k+1} U_{k(n-1)} \text { and } V_{k(n+1)}=V_{k} V_{k n}+(-1)^{k+1} V_{k(n-1)},
$$

where the initial conditions of the sequences $\left\{U_{k n}\right\}$ and $\left\{V_{k n}\right\}$ are $0, U_{k}$, and $2, V_{k}$, respectively. The Binet formulae are

$$
U_{k n}=\frac{\alpha^{k n}-\beta^{k n}}{\alpha-\beta} \text { and } V_{k n}=\alpha^{k n}+\beta^{k n}
$$

respectively.

Dual numbers were introduced in the 19th century by Clifford as a tool for his geometrical investigations [5] and provided us with a powerful tool for the analysis of complex numbers. It was used dual numbers and dual vectors on line geometry and kinematics $[4,16,17]$. Also, in [17], the geometric sense of dual numbers were studied in detail. Recently, dual numbers have been the subject of many studies especially in kinematics, robotic technology, quantum physics and mechanics. The dual numbers extend to the real numbers has the form

$$
d=a+\varepsilon a^{*},
$$

where $\varepsilon$ is the dual unit and $\varepsilon^{2}=0$ and $\varepsilon \neq 0$. In [13], Horadam introduced Fibonacci and Lucas quaternions. Many works have related to these quaternions considered by several authors (see, $[1-3,11,12,14,15])$. In [6], A. Cohen et al. showed that there were some applications of the "automatic differrentiation" property of the dual numbers in kinematics.

Hyper-dual numbers are a larger dimensional extension of dual numbers in a similar way that the quaternions are a larger dimensional extension of ordinary complex numbers. Firstly, J. A. Fike et al. introduced hyper-dual numbers in [8-10]. Hyper-dual numbers were defined like dual numbers but with the following difference: a hyper-dual number has four components unlike a dual number that has two components.

A hyper-dual number $\widetilde{x}=\left(x_{1}, x_{2}, x_{3}, x_{4}\right)$ and two dual units $\varepsilon_{1}, \varepsilon_{2}$, as follows:

$$
\widetilde{x}=x_{1}+x_{2} \varepsilon_{1}+x_{3} \varepsilon_{2}+x_{4} \varepsilon_{1} \varepsilon_{2},
$$

where

$$
\varepsilon_{1}^{2}=\varepsilon_{2}^{2}=\left(\varepsilon_{1} \varepsilon_{2}\right)^{2}=0
$$

and

$$
\varepsilon_{1}, \varepsilon_{2}, \varepsilon_{1} \varepsilon_{2} \neq 0 \text {. }
$$

The algebra of hyper-dual numbers is a ring with the following addition and multiplication operations:

$$
\begin{gathered}
\widetilde{x}+\widetilde{y}=x_{1}+y_{1}+\left(x_{2}+y_{2}\right) \varepsilon_{1}+\left(x_{3}+y_{3}\right) \varepsilon_{2}+\left(x_{4}+y_{4}\right) \varepsilon_{1} \varepsilon_{2}, \\
\widetilde{x} * \widetilde{y}=x_{1} y_{1}+\left(x_{1} y_{2}+x_{2} y_{1}\right) \varepsilon_{1}+\left(x_{1} y_{3}+x_{3} y_{1}\right) \varepsilon_{2} \\
+\left(x_{1} y_{4}+x_{2} y_{3}+x_{3} y_{2}+x_{4} y_{1}\right) \varepsilon_{1} \varepsilon_{2} .
\end{gathered}
$$


From these definitions, other mathematical operations can be defined, such as the multiplicative inverse of $\widetilde{x}$ :

$$
\frac{1}{\widetilde{x}}=\frac{1}{x_{1}}-\frac{x_{2}}{x_{1}^{2}} \varepsilon_{1}-\frac{x_{3}}{x_{1}^{2}} \varepsilon_{2}+\left(\frac{2 x_{2} x_{3}}{x_{1}^{3}}-\frac{x_{4}}{x_{1}^{2}}\right) \varepsilon_{1} \varepsilon_{2} .
$$

Hyper-dual numbers can be used to compute exact first- and second-order derivatives to evaluate gradients and Hessians for optimization methods. For $d=h_{1} \varepsilon_{1}+h_{2} \varepsilon_{2}+0 \varepsilon_{1} \varepsilon_{2}$, the Taylor series becomes

$$
f(x+d)=f(x)+h_{1} f^{\prime}(x) \varepsilon_{1}+h_{2} f^{\prime}(x) \varepsilon_{2}+h_{1} h_{2} f^{\prime \prime}(x) \varepsilon_{1} \varepsilon_{2} .
$$

In [5], A. Cohen et al. augmented the hyper-dual units in (2), as a combination of two "simple" dual numbers, to read as follows:

$$
\begin{aligned}
\varepsilon & =\varepsilon_{1} ; \quad \varepsilon^{*}=\varepsilon_{2}, \\
\varepsilon^{2} & =\left(\varepsilon^{*}\right)^{2}=\left(\varepsilon \varepsilon^{*}\right)^{2}=0, \\
\varepsilon, \varepsilon^{*}, \varepsilon \varepsilon^{*} & \neq 0 .
\end{aligned}
$$

Now, the equation in (1) can be written as the follows:

$$
\widetilde{x}=\left(x_{1}+x_{2} \varepsilon_{1}\right)+\varepsilon^{*}\left(x_{3}+x_{4} \varepsilon_{1}\right) .
$$

In the present paper, we define hyper-dual generalized Fibonacci numbers and give the Binet formulae, the generating functions. Moreover, we obtain some basic identities for these numbers.

\section{Hyper-dual generalized Fibonacci numbers}

In this section, we define hyper-dual generalized Fibonacci and Lucas numbers and give basic properties of them.

Definition 1. Hyper-dual generalized Fibonacci and Lucas numbers are defined by

$$
\widetilde{U}_{k n}=U_{k n}+U_{k(n+1)} \varepsilon_{1}+U_{k(n+2)} \varepsilon_{2}+U_{k(n+3)} \varepsilon_{1} \varepsilon_{2},
$$

and

$$
\widetilde{V}_{k n}=V_{k n}+V_{k(n+1)} \varepsilon_{1}+V_{k(n+2)} \varepsilon_{2}+V_{k(n+3)} \varepsilon_{1} \varepsilon_{2},
$$

respectively, where $\left\{U_{k n}\right\}$ and $\left\{V_{k n}\right\}$ are as above.

Now, we will give some properties of the hyper-dual generalized Fibonacci and Lucas numbers.

Theorem 2.1. Let $\widetilde{U}_{k n}$ and $\widetilde{V}_{k n}$ be hyper-dual generalized Fibonacci and Lucas numbers, respectively. The Binet formulae for these numbers have the forms

$$
\widetilde{U}_{k n}=\frac{\bar{\alpha} \alpha^{k n}-\bar{\beta} \beta^{k n}}{\alpha-\beta}
$$

and

$$
\widetilde{V}_{k n}=\bar{\alpha} \alpha^{k n}+\bar{\beta} \beta^{k n},
$$

where $\bar{\alpha}=1+\alpha^{k} \varepsilon_{1}+\alpha^{2 k} \varepsilon_{2}+\alpha^{3 k} \varepsilon_{1} \varepsilon_{2}$ and $\bar{\beta}=1+\beta^{k} \varepsilon_{1}+\beta^{2 k} \varepsilon_{2}+\beta^{3 k} \varepsilon_{1} \varepsilon_{2}$. 
Proof. By the Binet formula for $\left\{U_{k n}\right\}$, we have

$$
\begin{aligned}
& \widetilde{U}_{k n} \\
= & U_{k n}+U_{k(n+1)} \varepsilon_{1}+U_{k(n+2)} \varepsilon_{2}+U_{k(n+3)} \varepsilon_{1} \varepsilon_{2} \\
= & \frac{\alpha^{k n}-\beta^{k n}}{\alpha-\beta}+\frac{\alpha^{k(n+1)}-\beta^{k(n+1)}}{\alpha-\beta} \varepsilon_{1}+\frac{\alpha^{k(n+2)}-\beta^{k(n+2)}}{\alpha-\beta} \varepsilon_{2} \\
& +\frac{\alpha^{k(n+3)}-\beta^{k(n+3)}}{\alpha-\beta} \varepsilon_{1} \varepsilon_{2} \\
= & \frac{\left(1+\alpha^{k} \varepsilon_{1}+\alpha^{2 k} \varepsilon_{2}+\alpha^{3 k} \varepsilon_{1} \varepsilon_{2}\right) \alpha^{k n}-\left(1+\beta^{k} \varepsilon_{1}+\beta^{2 k} \varepsilon_{2}+\beta^{3 k} \varepsilon_{1} \varepsilon_{2}\right) \beta^{k n}}{\alpha-\beta} .
\end{aligned}
$$

We complete the proof. Similarly, the other result can be obtained.

Lemma 2.2. For $n, m \in \mathbb{Z}$, then

$$
\begin{aligned}
U_{k(n+m)}+(-1)^{k m+1} U_{k(n-m)} & =V_{k n} U_{k m}, \\
V_{k(n+m)}+(-1)^{k m+1} V_{k(n-m)} & =\Delta U_{k n} U_{k m},
\end{aligned}
$$

where $\Delta=V_{k}^{2}+4(-1)^{k+1}$.

Theorem 2.3. Let $\widetilde{U}_{k n}$ and $\widetilde{V}_{k n}$ be hyper-dual generalized Fibonacci and Lucas numbers, respectively. We have the following identities;

$$
\begin{gathered}
\widetilde{U}_{k(n+2)}=V_{k} \widetilde{U}_{k(n+1)}+(-1)^{k+1} \widetilde{U}_{k n}, \\
\widetilde{V}_{k(n+2)}=V_{k} \widetilde{V}_{k(n+1)}+(-1)^{k+1} \widetilde{V}_{k n}, \\
\widetilde{U}_{-k n}=(-1)^{k n+1}\left(\widetilde{U}_{k n}-V_{k n} \widetilde{U}_{0}\right), \\
\widetilde{V}_{-k n}=(-1)^{k n}\left(\widetilde{V}_{k n}-\Delta U_{k n} \widetilde{U}_{0}\right), \\
\widetilde{U}_{k(n+m)}+(-1)^{k m+1} \widetilde{U}_{k(n-m)}=U_{k m} \widetilde{V}_{k n},
\end{gathered}
$$

and

$$
\widetilde{V}_{k(n+m)}+(-1)^{k m+1} \widetilde{V}_{k(n-m)}=\Delta U_{k m} \widetilde{U}_{k n} .
$$

Proof. We will give the proof of (6). Using the Binet formula for the hyper-dual generalized Fibonacci number $\widetilde{U}_{k n}$ and $\alpha^{k n} \beta^{k n}=(-1)^{k n}$, we have

$$
\widetilde{U}_{-k n}=\frac{\bar{\alpha} \alpha^{-k n}-\bar{\beta} \beta^{-k n}}{\alpha-\beta}=(-1)^{k n} \frac{\bar{\alpha} \beta^{k n}-\bar{\beta} \alpha^{k n}}{\alpha-\beta} .
$$

Since $\bar{\alpha}=1+\alpha^{k} \varepsilon_{1}+\alpha^{2 k} \varepsilon_{2}+\alpha^{3 k} \varepsilon_{1} \varepsilon_{2}$ and $\bar{\beta}=1+\beta^{k} \varepsilon_{1}+\beta^{2 k} \varepsilon_{2}+\beta^{3 k} \varepsilon_{1} \varepsilon_{2}$, we get 


$$
\begin{aligned}
& \widetilde{U}_{-k n} \\
= & \frac{(-1)^{k n}}{\alpha-\beta}\left(\left(1+(-1)^{k} \beta^{-k} \varepsilon_{1}+\beta^{-2 k} \varepsilon_{2}+(-1)^{k} \beta^{-3 k} \varepsilon_{1} \varepsilon_{2}\right) \beta^{k n}\right. \\
& \left.-\left(1+(-1)^{k} \alpha^{-k} \varepsilon_{1}+\alpha^{-2 k} \varepsilon_{2}+(-1)^{k} \alpha^{-3 k} \varepsilon_{1} \varepsilon_{2}\right) \alpha^{k n}\right) \\
= & (-1)^{k n+1}\left(U_{k n}+(-1)^{k} U_{k(n-1)} \varepsilon_{1}+U_{k(n-2)} \varepsilon_{2}+(-1)^{k} U_{k(n-3)} \varepsilon_{1} \varepsilon_{2}\right) \\
= & (-1)^{k n+1}\left(\widetilde{U}_{k n}-\left(U_{k(n+1)}-(-1)^{k} U_{k(n-1)}\right) \varepsilon_{1}-\left(U_{k(n+2)}-U_{k(n-2)}\right) \varepsilon_{2}\right. \\
& \left.-\left(U_{k(n+3)}-(-1)^{k} U_{k(n-3)}\right) \varepsilon_{1} \varepsilon_{2}\right) .
\end{aligned}
$$

By (3), we get

$$
\begin{aligned}
& \widetilde{U}_{-k n}=(-1)^{k n+1}\left(\widetilde{U}_{k n}-U_{k} V_{k n} \varepsilon_{1}-U_{2 k} V_{k n} \varepsilon_{2}-U_{3 k} V_{k n} \varepsilon_{1} \varepsilon_{2}\right) \\
= & (-1)^{k n+1}\left(\widetilde{U}_{k n}-V_{k n}\left(U_{0}+U_{k} \varepsilon_{1}+U_{2 k} \varepsilon_{2}+U_{3 k} \varepsilon_{1} \varepsilon_{2}\right)\right) \\
= & (-1)^{k n+1}\left(\widetilde{U}_{k n}-V_{k n} \widetilde{U}_{0}\right) .
\end{aligned}
$$

Thus, the desired result is obtained. Similarly, the proofs of the other results are given.

Theorem 2.4. The generation functions for hyper-dual generalized Fibonacci and Lucas numbers $\widetilde{U}_{k n}$ and $\widetilde{V}_{k n}$ are

$$
g(x)=\frac{\widetilde{U}_{0}+\left(\widetilde{U}_{k}-\widetilde{U}_{0} V_{k}\right) x}{1-V_{k} x+(-1)^{k} x^{2}} \text { and } h(x)=\frac{\widetilde{V}_{0}+\left(\widetilde{V}_{k}-\widetilde{V}_{0} V_{k}\right) x}{1-V_{k} x+(-1)^{k} x^{2}},
$$

respectively.

Proof. Let

$$
g(x)=\widetilde{U}_{0}+\widetilde{U}_{k} x+\widetilde{U}_{2 k} x^{2}+\cdots+\widetilde{U}_{k n} x^{n}+\cdots
$$

be the generating function of the hyper-dual generalized Fibonacci number $\widetilde{U}_{k n}$. Since the orders of $\widetilde{U}_{k(n-1)}$ and $\widetilde{U}_{k(n-2)}$ are 1 and 2 less than the order of $\widetilde{U}_{k n}$, respectively and (4), we have $x g(x)$ and $x^{2} g(x)$ :

$$
\begin{aligned}
V_{k} x g(x) & =\widetilde{U}_{0} V_{k} x+\widetilde{U}_{k} V_{k} x^{2}+\widetilde{U}_{2 k} V_{k} x^{3}+\ldots \widetilde{U}_{k(n-1)} V_{k} x^{n}+\ldots \\
(-1)^{k} x^{2} g(x) & =\widetilde{U}_{0}(-1)^{k} x^{2}+\widetilde{U}_{k}(-1)^{k} x^{3}+\ldots \widetilde{U}_{k(n-2)}(-1)^{k} x^{n}+\ldots
\end{aligned}
$$

Then we have

$$
\left(1-V_{k} x+(-1)^{k} x^{2}\right) g(x)=\widetilde{U}_{0}+\left(\widetilde{U}_{k}-\widetilde{U}_{0} V_{k}\right) x
$$

Thus, the proof is complete. Similarly, by (5), the other generating function is obtained.

Theorem 2.5. Let $\widetilde{U}_{k n}$ and $\widetilde{V}_{k n}$ be hyper-dual generalized Fibonacci and Lucas numbers, respectively. For $m, n, r \in \mathbb{Z}$,

$$
\begin{aligned}
& \widetilde{U}_{k(n+r)} \widetilde{U}_{k m}-\widetilde{U}_{k n} \widetilde{U}_{k(m+r)}=(-1)^{k n} \frac{U_{k r} U_{k(m-n)}}{4}\left(\widetilde{V}_{0}^{2}-\Delta{\widetilde{U_{0}}}^{2}\right), \\
& \widetilde{V}_{k(n+r)} \widetilde{V}_{k m}-\widetilde{V}_{k n} \widetilde{V}_{k(m+r)}=(-1)^{k n+1} \frac{\Delta U_{k r} U_{k(m-n)}}{4}\left(\widetilde{V}_{0}{ }^{2}-\Delta{\widetilde{U_{0}}}^{2}\right),
\end{aligned}
$$


and

$$
\widetilde{U}_{k(n+r)} \widetilde{V}_{k m}-\widetilde{V}_{k n} \widetilde{U}_{k(m+r)}=(-1)^{k n+1} \frac{V_{k r} U_{k(m-n)}}{4}\left(\widetilde{V}_{0}^{2}-\Delta{\widetilde{U_{0}}}^{2}\right),
$$

where $\left\{U_{k n}\right\}$ and $\left\{V_{k n}\right\}$ are as above.

Proof. By Binet formula for the hyper-dual generalized Fibonacci number $\widetilde{U}_{k n}$, we have

$$
\begin{aligned}
& \widetilde{U}_{k(n+r)} \widetilde{U}_{k m}-\widetilde{U}_{k n} \widetilde{U}_{k(m+r)} \\
= & \frac{\bar{\alpha} \alpha^{k(n+r)}-\bar{\beta} \beta^{k(n+r)} \bar{\alpha} \alpha^{k m}-\bar{\beta} \beta^{k m}}{\alpha-\beta}-\frac{\bar{\alpha} \alpha^{k n}-\bar{\beta} \beta^{k n}}{\alpha-\beta} \frac{\bar{\alpha} \alpha^{k(m+r)}-\bar{\beta} \beta^{k(m+r)}}{\alpha-\beta} \\
= & \frac{1}{(\alpha-\beta)^{2}}\left\{\bar{\alpha}^{2} \alpha^{k(m+n+r)}-\bar{\alpha} \bar{\beta} \alpha^{k(n+r)} \beta^{k m}-\bar{\alpha} \bar{\beta} \beta^{k(n+r)} \alpha^{k m}+\bar{\beta}^{2} \beta^{k(m+n+r)}\right. \\
& \left.-\bar{\alpha}^{2} \alpha^{k(m+n+r)}+\bar{\alpha} \bar{\beta} \alpha^{k(m+r)} \beta^{k n}+\bar{\alpha} \bar{\beta} \alpha^{k n} \beta^{k(m+r)}-\bar{\beta}^{2} \beta^{k(m+n+r)}\right\} .
\end{aligned}
$$

Using the equality $\alpha^{k n} \beta^{k n}=(-1)^{k n}$, we write

$$
\begin{aligned}
\widetilde{U}_{k(n+r)} \widetilde{U}_{k m}-\widetilde{U}_{k n} \widetilde{U}_{k(m+r)} & =\frac{\bar{\alpha} \bar{\beta}}{(\alpha-\beta)^{2}}\left(\alpha^{k r}-\beta^{k r}\right)\left(\alpha^{k m} \beta^{k n}-\alpha^{k n} \beta^{k m}\right) \\
& =\frac{(-1)^{k n} \bar{\alpha} \bar{\beta}}{(\alpha-\beta)^{2}}\left(\alpha^{k r}-\beta^{k r}\right)\left(\alpha^{k(m-n)}-\beta^{k(m-n)}\right) .
\end{aligned}
$$

Since $\bar{\alpha} \bar{\beta}=\frac{{\widetilde{V_{0}}}^{2}-\Delta{\widetilde{U_{0}}}^{2}}{4}$ and Binet formula of the sequence $\left\{U_{k n}\right\}$, we have

$$
\widetilde{U}_{k(n+r)} \widetilde{U}_{k m}-\widetilde{U}_{k n} \widetilde{U}_{k(m+r)}=(-1)^{k n} \frac{U_{k r} U_{k(m-n)}}{4}\left(\widetilde{V}_{0}^{2}-\Delta{\widetilde{U_{0}}}^{2}\right)
$$

which completes the proof. Similarly, the other results are given.

When $r=1$ in (7), we have the following conclusion.

Corollary 2.5.1 (d'Ocagne's Identity). Let $\widetilde{U}_{k n}$ be a hyper-dual generalized Fibonacci number. For $m, n \in \mathbb{Z}$,

$$
\widetilde{U}_{k(n+1)} \widetilde{U}_{k m}-\widetilde{U}_{k n} \widetilde{U}_{k(m+1)}=(-1)^{k n} \frac{U_{k} U_{k(m-n)}}{4}\left(\widetilde{V}_{0}^{2}-\Delta{\widetilde{U_{0}}}^{2}\right) .
$$

Taking $n, n-r$ instead of $m, n$ in (7), respectively, we have the following conclusion.

Corollary 2.5.2 (Catalan's Identity). Let $\widetilde{U}_{k n}$ be a hyper-dual generalized Fibonacci number. For $n, r \in \mathbb{Z}$,

$$
\widetilde{U}_{k n}^{2}-\widetilde{U}_{k(n-r)} \widetilde{U}_{k(n+r)}=(-1)^{k(n-r)} \frac{U_{k r}^{2}}{4}\left(\widetilde{V}^{2}-\Delta{\widetilde{U_{0}}}^{2}\right) .
$$

Taking $n-1$ instead of $m$ and $r=1$ in (7), we have the following conclusion.

Corollary 2.5.3 (Cassini Identity). Let $\widetilde{U}_{k n}$ be a hyper-dual generalized Fibonacci number. For $n \in \mathbb{Z}$,

$$
\widetilde{U}_{k(n+1)} \widetilde{U}_{k(n-1)}-\widetilde{U}_{k n}^{2}=(-1)^{k n+k+1} \frac{U_{k}^{2}}{4}\left(\widetilde{V}_{0}^{2}-\Delta{\widetilde{U_{0}}}^{2}\right)
$$


Theorem 2.6. Let $\widetilde{U}_{k n}$ and $\widetilde{V}_{k n}$ be hyper-dual generalized Fibonacci and Lucas numbers, respectively. Then

$$
\begin{aligned}
\sum_{i=0}^{n} \widetilde{U}_{k(a i+b)} & =\frac{\widetilde{U}_{k b}-(-1)^{k a} \widetilde{U}_{k(b-a)}-\widetilde{U}_{k(a(n+1)+b)}+(-1)^{k a} \widetilde{U}_{k(a n+b)}}{1-V_{k a}+(-1)^{k a}} \\
\sum_{i=0}^{n} \widetilde{V}_{k(a i+b)} & =\frac{\widetilde{V}_{k b}-(-1)^{k a} \widetilde{V}_{k(b-a)}-\widetilde{V}_{k(a(n+1)+b)}+(-1)^{k a} \widetilde{V}_{k(a n+b)}}{1-V_{k a}+(-1)^{k a}}
\end{aligned}
$$

Proof. Observe that

$$
\begin{aligned}
& \sum_{i=0}^{n} \widetilde{U}_{k(a i+b)}=\sum_{i=0}^{n} \frac{\bar{\alpha} \alpha^{k(a i+b)}-\bar{\beta} \beta^{k(a i+b)}}{\alpha-\beta} \\
= & \frac{1}{\alpha-\beta}\left(\bar{\alpha} \alpha^{k b} \sum_{i=0}^{n} \alpha^{k a i}-\bar{\beta} \beta^{k b} \sum_{i=0}^{n} \beta^{k a i}\right) \\
= & \frac{1}{\alpha-\beta}\left(\bar{\alpha} \alpha^{k b} \frac{1-\alpha^{k a(n+1)}}{1-\alpha^{k a}}-\bar{\beta} \beta^{k b} \frac{1-\beta^{k a(n+1)}}{1-\beta^{k a}}\right) \\
= & \frac{1}{\alpha-\beta}\left\{\frac{\bar{\alpha} \alpha^{k b}\left(1-\beta^{k a}-\alpha^{k a(n+1)}+\beta^{k a} \alpha^{k a(n+1)}\right)}{1-V_{k a}+(-1)^{k a}}\right. \\
& \left.-\frac{\bar{\beta} \beta^{k b}\left(1-\alpha^{k a}-\beta^{k a(n+1)}+\alpha^{k a} \beta^{k a(n+1)}\right)}{1-V_{k a}+(-1)^{k a}}\right\} .
\end{aligned}
$$

By definition of hyper-dual generalized Fibonacci numbers $\widetilde{U}_{k n}$, we write

$$
\sum_{i=0}^{n} \widetilde{U}_{k(a i+b)}=\frac{\widetilde{U}_{k b}-(-1)^{k a} \widetilde{U}_{k(b-a)}-\widetilde{U}_{k(a(n+1)+b)}+(-1)^{k a} \widetilde{U}_{k(a n+b)}}{1-V_{k a}+(-1)^{k a}},
$$

which completes the proof. Similarly, the desired result is obtained.

\section{References}

[1] Akkus, I., \& Keçilioğlu, O. (2015). Split Fibonacci and Lucas octonions, Adv. Appl. Clifford Algebras, 25 (3), 517-525.

[2] Keçilioğlu, O., \& Akkus, I. (2015). The Fibonacci octonions, Adv. Appl. Clifford Algebras, 25 (1), 151-158.

[3] Akyiğit, M., Köksal, H. H., \& Tosun, M. (2014). Fibonacci generalized quaternions, Adv. Appl. Clifford Algebras, 24, 535-545.

[4] Clifford, W. K. (1873). Preliminary sketch of bi-quaternions, Proceeding of London Mathematical Society, 4 (64), 65, 361-395.

[5] Cohen, A., \& Shoham, M. (2017). Application of hyper-dual numbers to rigid bodies equations of motion, Mechanism and Machine Theory, 111, 76-84. 
[6] Cohen, A., \& Shoham, M. Application of hyper-dual numbers to multi-body kinematics, ASME J. Mech. Robot, doi:10.1115/1.4030588.

[7] Kılıç, E., \& Stanica, P. (2009). Factorizations and representations of second order linear recurrences with indices in arithmetic progressions, Bulletin of the Mexican Mathematical Society, 15 (1), 23-36.

[8] Fike, J. A. (2009). Numerically exact derivative calculations using hyper-dual numbers, 3rd Annural Student Joint Workshop in Simulation-Based Engineering and Design, 18 June 2009.

[9] Fike, J. A., \& Alonso, J. J. (2011). The development of hyper-dual numbers for exact second-derivative calculations, 49th AIAA Aerospace Sciences Meeting, 4-7 January 2011, Article No. AIAA 2011-886, 17 pages.

[10] Fike, J. A., Jongsma, S., \& Alonso, J. J. (2011). E van der Weida, Optimization with gradient and Hessian information calculated using hyper-dual numbers, 29 AIAA Applied Aerodynamics Conference, Honolulu, Article No. AIAA 2011-3807, 19 pages.

[11] Akkus, I., \& Kızılaslan, G. (2018). On some properties of Tribonacci quaternions, An. Stiint. Univ. Ovidius Constanta Ser. Mat., 26 (3), 5-20.

[12] Halıc1, S. (2015). On Fibonacci quarternions, Adv. Appl. Clifford Algebras, 25, 577-590.

[13] Horadam, A. F. (1963). Complex Fibonacci numbers and Fibonacci quaternions, American Math. Monthly, 70, 289-291.

[14] Iyer, M. R. (1969.) Some results on Fibonacci quaternions, The Fibonacci Quart., 7 (2), 201-210.

[15] Nurkan, S. K., \& Güven, I. A. (2015). Dual Fibonacci quaternions, Adv. Appl. Clifford Algebras, 25, 403-414.

[16] Veldkamp, G. R. (1976). On the use of dual numbers, vectors and matrices in instantaneous, spatial kinematics, Mechanism and Machine Theory, 11 (2), 141-156.

[17] Yaglom, I. M. (1979). A Simple Non-Euclidean Geometry and Its Physical Basis, SpringerVerlag, New York. 